\title{
Analysing bullwhip effect in supply networks under information sharing and exogenous uncertainty
}

\author{
Mehdi Seifbarghy, Mitra Darvish and Fatemeh Akbari \\ Department of Industrial Engineering, Alzahra University, Tehran, Iran
}

\begin{abstract}
This paper analyses the bullwhip effect in single product supply network topologies, operated with linear and time-invariant inventory management policies and shared supply network information, considering exogenous uncertainty. Information sharing is determined as the degree of coordination across the supply network. Exogenous uncertainty (e.g., transportation delay) cannot be governed by any supply network members. We characterise the stream of orders placed at any stage of the network assuming the customer demand is ergodic. In fact, this paper gives exact formulae to predict the magnitude of bullwhip effect in any shared supply network information topologies. The mentioned formulae is explored by means of mathematical method called frequency domain analysis (FDA) and the relevant analyses are progressed by Fourier transform method. The main contribution of the present work is defined as considering information sharing and exogenous uncertainty simultaneously in supply networks and using Fourier transforms.
\end{abstract}

Keywords: Bullwhip effect (BWE), Supply networks, Information sharing, Frequency domain analysis (FDA), Exogenous uncertainty. 